\title{
Quantum Nature of Distortion and Delay of Satellite Signals II
}

\author{
Gennady V. Golubkov ${ }^{1,2}$, Michael I. Manzhelii1, Lev V. Eppelbaum ${ }^{3 *}$ \\ ${ }^{1}$ Semenov Institute of Chemical Physics of RAS, Moscow, Russia \\ ${ }^{2}$ National Research Center "Kurchatov Institute", Moscow, Russia \\ ${ }^{3}$ Department of Earth Sciences, Tel Aviv University, Tel Aviv, Israel \\ Email: kolupanovo@gmail.com, mike.manzheliy@gmail.com, *levap@post.tau.ac.il
}

How to cite this paper: Golubkov, G.V., Manzhelii, M.I. and Eppelbaum, L.V. (2018) Quantum Nature of Distortion and Delay of Satellite Signals II. Positioning, 9, 47-72. https://doi.org/10.4236/pos.2018.93004

Received: July 18, 2018

Accepted: August 14, 2018

Published: August 17, 2018

Copyright (C) 2018 by authors and Scientific Research Publishing Inc. This work is licensed under the Creative Commons Attribution International License (CC BY 4.0).

http://creativecommons.org/licenses/by/4.0/

\begin{abstract}
A detailed analysis of the influence of Rydberg states to the behavior of GPS satellite signals in the D and $\mathrm{E}$ atmospheric layers has been carried out. It is demonstrated that these states are the main reason for the GPS signal distortion. It is shown that the behavior of satellite signals is associated with the spectral characteristics of the UHF radiation of the Rydberg states depending on the geomagnetic conditions of ionosphere. The foundations of the quantum theory of distortion and delay of GPS satellite signal propagation through $\mathrm{D}$ and $\mathrm{E}$ atmospheric layers are analyzed and expounded. The problem reduces to the resonant scattering of photons, moving in the electromagnetic field of the signal, to the Rydberg complexes populated in a two-temperature non-equilibrium plasma. The processes of creation of additional photons because of stimulated emission and resonance scattering of photons are considered. In the present work, the quantum theory of the propagation of a satellite signal in the Earth's upper atmosphere, firstly earlier proposed by the same authors, is described in detail. The general problems of the theory and possible theoretical and applied consequences are discussed. It is explained that two main processes occurring here, are directly related to the resonant quantum properties of the propagation medium. The first process leads to a direct increase in the power of the received signal, and second-to a shift in the signal carrier frequency $\Delta v$ and the time delay $\Delta \tau$ of its propagation. The main reasons of the processes are scattering of the Rydberg electron by the ion core and presence of the neutral medium molecule in the intermediate autoionization states of the composite system populated by the strong non-adiabatic coupling of electron and nuclear motions. The main purposes of our investigation are the physical justification of the formation of parameters $\Delta v$ and $\Delta \tau$ using the quantum dynamics of the electron behavior in the intermediate state of the Rydberg complex $A^{* *} M$ and the esti-
\end{abstract}


mation of the quantities of $\Delta v$ and $\Delta \tau$ in the elementary act of elastic (Rayleigh) photon scattering.

\section{Keywords}

$\mathrm{D}$ and E Atmospheric Layers, 1 Mixing, Rydberg Complex, Satellite Radio Signal, Electromagnetic Field, Resonant Photon Scattering, Distortion and Delay of Satellite Signal

\section{Introduction}

The GPS system is employed now as a highly important element of the global information infrastructure [1]. Free and reliable access to this system led to the development of hundreds of applications that affects majority aspects of the modern infrastructure, environment, science, medicine, etc.

GPS technology is now used almost everywhere, from precise clocks and cell phones, to bulldozers, sea vessels, military applications and bank computers. It increases productivity in various sectors of the economy, including agriculture, aviation, building, defense systems, all branches of the Earth's sciences, sport applications, postal service, etc. The use of the GPS system saves lives, prevents traffic accidents, and promotes search and rescue operations, speeds up the delivery of emergency services and the elimination of the consequences of natural disasters. This system is vital for the next generation of the automatic transportation [2], which should help to improve vehicle safety with increasing reliability and capacity.

The GPS system is also of high importance for the majority of scientific, environmental, technical and medical tasks: weather (climate) forecasting e.g. [3], earthquake monitoring, searching economic minerals, environmental protection, archaeological prospection, and safe driving [4]-[11]. The GPS system (space sensor net) is also crucial for any national security.

However, GPS is not free from some disturbing effects. Spontaneous amplification of the solar activity accompanied by significant increase of electromagnetic radiation and ejection of high-energy particles leads to strong disturbances in the upper atmosphere and ionosphere of Earth. A presence of such failures in the frequency range of $1.2-1.6 \mathrm{GHz}$ is the one of the most significant manifestations of such perturbations in the GPS data. The increase in the solar activity leads to significant decrease in the signal/noise ratio and, as a result, to the partial inability to decode the messages contained in the signal. Careful analysis of literature sources does not give an understanding of this phenomenon. We propose that our theory presented in this study will elucidate the physical causes of the disturbing effect.

It was shown in [12] that during the solar flares of different power there was a certain sequence of decreasing carrier/noise ratio for the frequencies $L_{1}=$ $1.57542 \mathrm{GHz}$ and $L_{2}=1.22760 \mathrm{GHz}$ (Figure 1(a)). During the solar flare of X-1 
level (22.15 UTC, December 14, 2006) the carrier/noise ratio for the frequency $L_{1}$ had become worse. At the same time, the carrier/noise ratio for the $L_{2}$ frequency remained unchanged (see Figure 1(a)). The flare of the X-3 level (02.40 UT, December 13, 2006) led to a simultaneous deterioration of the carrier/noise ratio for both frequencies (Figure 1(b)). The duration of the phenomena observed in both cases was about 30 minutes. This behavior of signal/noise ratio in principle cannot be explained by the impact of the broadband solar radio emission.

More powerful geomagnetic disturbances lead to complete disappearance of the signal at the GPS receiver for a sufficiently long period of time [13]. For instance, the data obtained at the Sao Luis Observatory (Brazil) on September 15-16, 2011 showed the loss of GPS signal occurred several times during the day. The signal at the receiver sporadically disappeared five times for 5 - 30 minutes each between 16.00 UTC September 15 to 01.00 UTC September 16, 2011. Moreover, the horizontal positioning error during these days greatly exceeded the value of $50 \mathrm{~m}$.

The next phenomenon that deserves a comprehensive attention, is the increasing power of the GPS receiver signal during periods of strong solar activity.
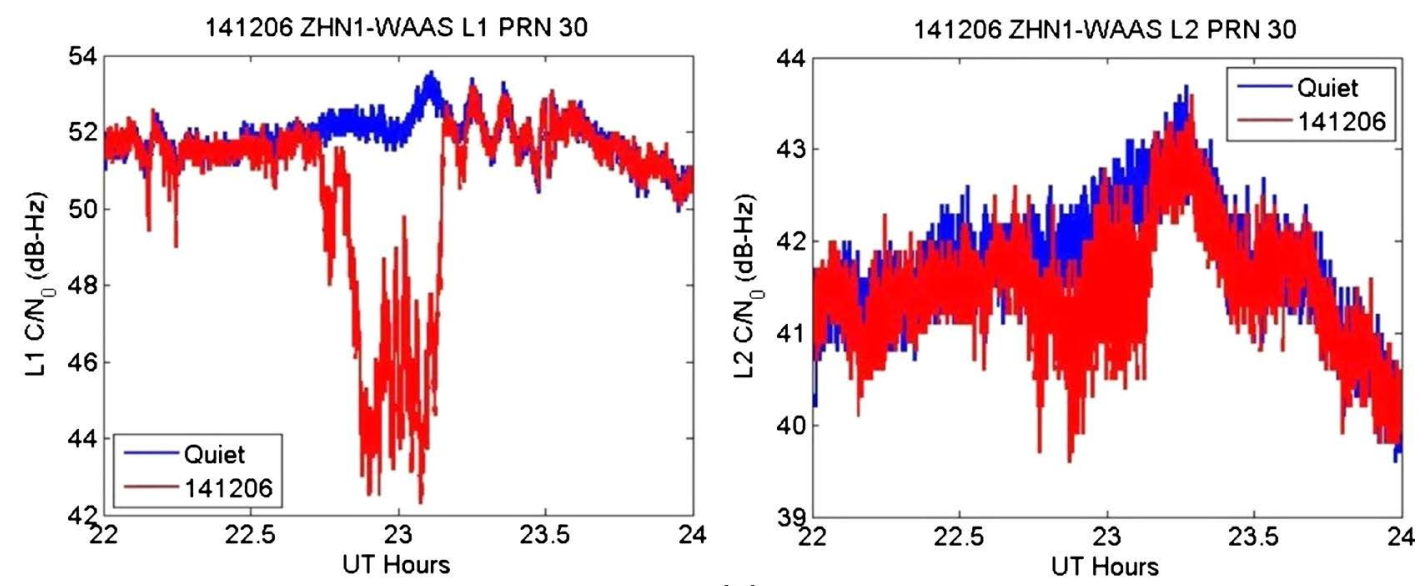

(a)
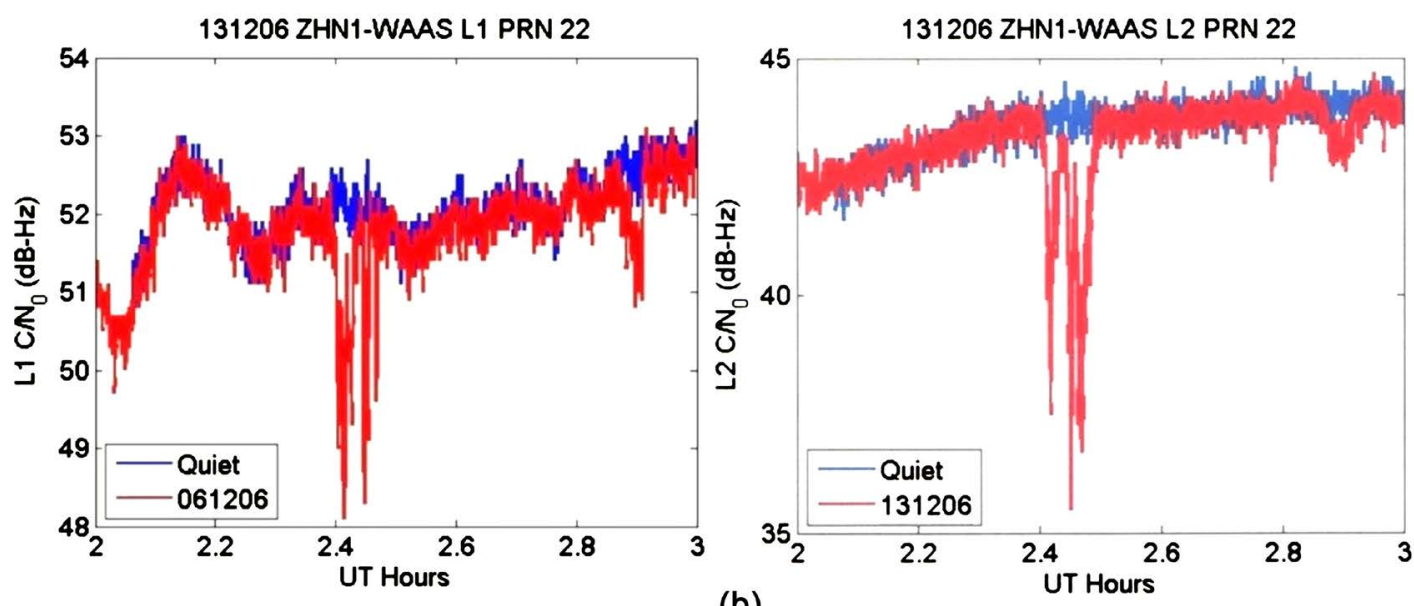

(b)

Figure 1. (a) The solar flare of class X-1 in December 14, 2006 at 22.15 UT; (b) The solar flare of class X-3 in December 13, 2006 at 02.40 UT. 
The time-dependence of the power of the GPS signal, and an integral number of failures at the receiver during the geomagnetic disturbance on July 15, 2000 are shown in Figure 2 (after [14]). The left vertical axis shows the intensity of the GPS signal in relative units. At the right axis the total number of failures is displayed. The whole process was observed during three hours. There was an increase of about three times in the intensity of the signal at the receiver $S(t)$ with respect to the satellite signal power. The integral number of failures grew with increasing intensity of the received signal, although it is obvious that the increase in the number of failures at high levels of carrier signal should not occur.

The authors [14] did not give an explanation for the growth of intensity. Indeed, it is difficult to assume that the signal increases over time caused by the presence of solar radio-wave radiation, since the GPS signal registered at the receiver is a correctly decoded signal from the satellite. The right-hand part of the graph is intermittent what reflects the lack of satellite data identification.

Effect of the solar activity bursts on the stability of the satellite navigation systems is well established in the numerous studies. However, the physical mechanisms of this effect have not received a satisfactory explanation. It was earlier assumed that the main contribution to the positioning error makes F-layer of the ionosphere, which determines the total electron content (TEC) in the atmosphere. However, the effects of the solar flares and solar activity most clearly and synchronously are manifested in the ionization of $\mathrm{D}$ and $\mathrm{E}$ ionospheric layers [15]. Experimental studies [16] [17] [18] have shown that the positioning errors are caused by the solar perturbation and arise due to changes in characteristics of the signals while passing through the lower part of $\mathrm{D}$ and $\mathrm{E}$ ionosphere layers. This fact is clearly demonstrated in Figure 3 where data from [16] were used.

Figure 3 displays the dependence of the average signal/noise ratio on the time of rocket flight, launched from Sweden to the ballistic orbit with a maximum of $700 \mathrm{~km}$. Based on the above experimental data, the following analysis is presented below. Despite the fact that the frequency spectrum of the solar radiation is uniform in the range from 2 to $18 \mathrm{GHz}$ [19], selective sequence of the signal/noise ratio reduction is observed for the frequencies $L_{1}$ and $L_{2}$ [20] under conditions of increased solar activity. Also let us note that in this paper is presented a similar, uniform by frequency, time relationship between the radiation power (in the range of $1.2-1.6 \mathrm{GHz}$ ) and the high solar activity. This means that

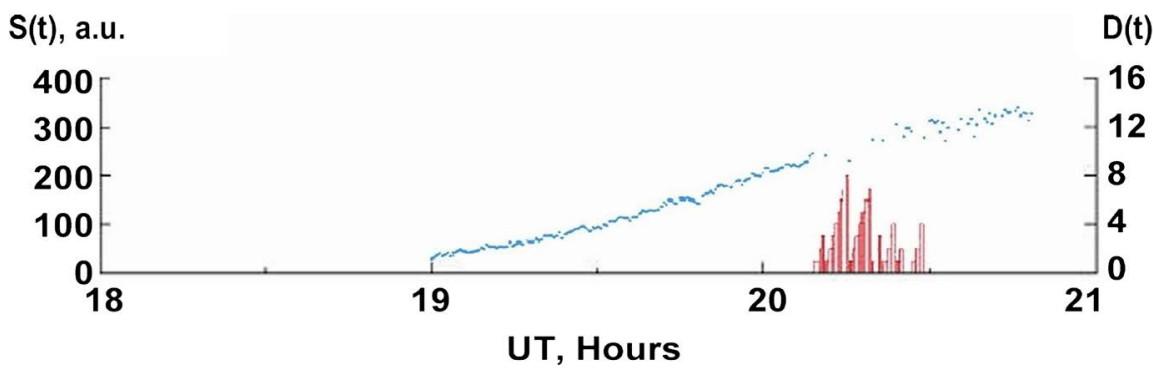

Figure 2. Dependence of the intensity $S(t)$ of the GPS signal registered at the receiver and the number of failures $D(t)$ per unit time (after [14]). 


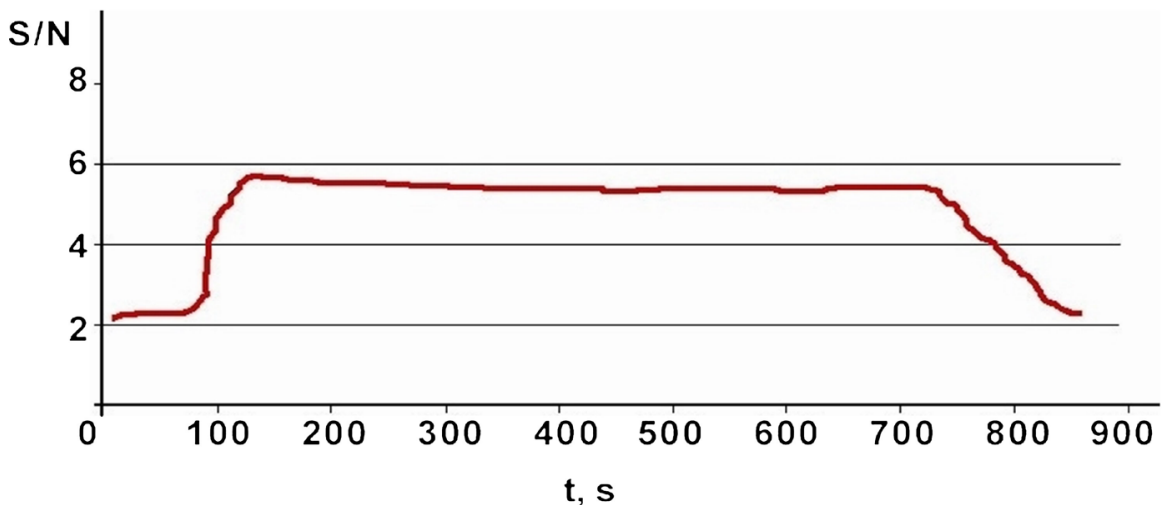

Figure 3. Dependence of the signal/noise ratio on time during rocket flight.

solar radiation cannot be responsible for this effect directly and the physical cause of the phenomenon lies elsewhere.

Furthermore, in Afraimovich's experiments [14], an increase of failures is observed with increasing intensity of the received signal, i.e. the received signal differs from the original one that cannot occur from the incoherent radiation of the Sun. Such a behavior of the signal can be explained by the fact that it is a subject of the resonance effects of the propagation medium, the nature of which is currently under intensive study [21]-[26].

Therefore, we have two obvious contradictions, which at first view are incompatible with each other. The first phenomenon is due to the incoherent light exposure, and the second phenomenon is caused by the coherent and resonant light influence. There is an essential theoretical problem to combine together these two phenomena. On the other hand, according to measurements [16] [17], the most powerful GPS signal distortion occurs in the lower part of the D and E ionospheric layers (below $110 \mathrm{~km}$ above the Earth's surface), the physical essence of which is discussed in detail below.

Thus, the results of investigations indicate that the solar activity disturbances are an important factor affecting the quality of GPS signal. Positioning errors in such periods can reach hundreds of meters at the Earth's surface that is high significant practically in all GPS applications. At the same time, the results of studies show that positioning errors for the infrastructure objects do not exceed several meters even in the conditions of geomagnetic disturbances [27]. The estimates of positioning errors in the geometric optics approximation, taking into account refraction on ionospheric inhomogeneities in the $\mathrm{F}$ domain of the ionosphere, also do not exceed several meters at the earth's surface [28]. Direct measurements using ballistic missiles [16] and the radio-occultation method [17] have shown that the atmospheric layer, where the signal mainly degrades, is located at the altitudes of $80-110 \mathrm{~km}$ above the Earth's surface, i.e. in the D and E layers of the atmosphere. When the satellite signal passes through these layers, multiple resonance scattering of photons occurs on the orbitally degenerate Rydberg complexes $A^{* *} M$, whose states are split by interaction with the neutral medium; between two last subjects occur the radiation transitions [26]. 
This paper essentially extends the materials firstly published by the same authors [29], where was shown that the processes occurring in the atmosphere are directly related to the resonant quantum properties of the satellite GPS signal propagation through Earth's atmosphere. We considered the processes of formation of additional photons as result of stimulated emission and resonance scattering of photons. The first process leads to an increase of the power of the received signal, and second provides a shift in the signal carrier frequency $\Delta v$ and the time delay $\Delta \tau$ of its propagation. This is due to the scattering of the Rydberg electron moving in the Rydberg complex $A^{* \star} \mathrm{M}$ on the ionic molecular core and the neutral molecule of the medium $\mathrm{M}$ in the intermediate autoionization states owing to the compound system populating by a strong nonadiabatic coupling of electron and nuclear motions. As a result, shift of the carrier frequency and time delay during GPS signal propagation through atmosphere are occurred.

The physical justification of formation of the shift $\Delta v$ and delay time $\Delta \tau$ of the satellite signal by the quantum dynamics of the electron behavior in the intermediate state of the Rydberg complex $A^{* *} M$ and the estimation of these quantities in the elementary act of elastic (Rayleigh) photon scattering are the main purposes of our investigation. A detailed description of the manifestation of the resonant quantum properties of the propagation medium of satellite GPS signals are presented in the first five sections of this paper. The influence of Rydberg states on these signals in the D and $\mathrm{E}$ layers of the atmosphere is analyzed. It is shown that the behavior of the signals is related to the spectral characteristics of the microwave radiation of Rydberg complex $A^{* *} M$ which depend on the geomagnetic state of the ionosphere. The basics of quantum theory of distortion and delay of the GPS signal are disputed in detailed in 'Discussion'. It is established that the delay time is completely determined by the dynamics of the intermediate autoionization states of the Rydberg molecules due to their interaction with the surrounding medium. Finally, the stages of the further construction of the general theory are formulated and discussed.

\section{Present State of the Theory and Experiments}

It is known that the signal propagation delay is observed when the satellite signals propagate through the ionosphere containing free electrons and positively charged ions [30]. The presence of electrons is confirmed by measurements carried out by ionosondes (high-frequency radars) located at the Earth. The signal received by the radar is a probing signal that has passed twice through the ionosphere and is reflected from the ionosphere F2 layer. The time delay of the signal passing through the ionosphere leads to serious limitations in the accuracy of measurement of the distance between the satellite and receiver (last is located at the Earth's surface). The accuracy of measuring the distance calculated by the propagation time of a signal depends on the density of free electrons in the Earth's ionosphere along the path of signal propagation. The magnitude of 
the signal propagation delay is usually described by the simple formula proposed in [31]

$$
\Delta \tau=\frac{40.3}{3 \times 10^{8} v^{2}} \int N \mathrm{~d} l,
$$

where $v$ is the frequency of the radio waves emitted by the satellite in $\mathrm{Hz}$, $\int N \mathrm{~d} l$ is the total electron content (TEC) in the signal propagation path, along the column cross-section for one $\mathrm{m}^{2}$.

According to the measurements performed by the ionosondes, the main role in the TEC forming has the ionosphere layer F2. The greatest contribution comes from the region located near the maximum of the distribution function of the electron density which is detected by the high-frequency pulse radars. Since such measurements are carried out, starting from 1930, there a rich statistical material has been accumulated. It allows using regular measurements in order to create a set of different statistics, and reconstruct on their basis a distribution of the average electron density in the F2 layer for seasonal time of day, geographic location, etc. The basis conception presented in [31] is the assumption that the Earth's ionosphere is a dispersive plasma medium. This representation is, strictly speaking, valid only for a homogeneous medium [32]. Klobuchar [31] methodology is used worldwide for the monitoring of ionosphere scintillation effects and is widely known as a "Klobuchar Model".

In essence, this is the simplest model of the ionosphere plasma, where the dispersion of medium is associated with the electron density variation at a fixed frequency. The difference between the results of the model calculations [32] and experiment gives an error of the time delay (see Equation (1)) up to 50\%. The measurement delay time error at the same frequency, e.g. $L_{1}$, can exceed $100 \mathrm{~ns}$ with respect to the TEC value. Note, that along with the model, there are many other models of the ionosphere based on statistical measurements, which differ from each other by the degree of complexity. However, their use does not allow completely reduce the measurement error of $\Delta \tau$. In fact, the best correction of ionosphere effects can be achieved here only by independent measurements strictly aligned with the direction of the GPS signal propagation from each GPS satellite to the receiver located at the ground surface. Thus, an analysis of results should be carried out without the statistical averages. This is especially concerns the velocity of variation of the group delay $\Delta \tau$.

At present, the physical interpretation of the relationship between the solar events and the disruption of the functioning of satellite navigation systems is inadequate and needs in additional examination. However, analysis of literature indicates that the main efforts of engineers and researchers are concentrated only for the improvement of the transmitting and receiving equipment of navigation systems without attempts to study the natural phenomena occurring in the Earth's atmosphere. We believe that the most problem is to find out which physical and chemical processes affect the propagation of radio waves in atmosphere at this frequency range [26]. 
An analysis of existing experimental data shows that the main contribution to positioning errors is formed on the sections of radio paths passing through the lower ionosphere. The validity of this conclusion is confirmed by numerous studies and, in particular [16] [17] [18], where was shown that in the daytime positioning errors are created mainly at the altitudes less than $110 \mathrm{~km}$. The influence of the lower ionosphere on the reception quality of GPS satellites is convincingly demonstrated in Figure 3. The dependence depicted in this figure corresponds to results obtained at satellite which during the entire flight proved to be practically in an upright position. In addition, in Afraimovich's experiment [14] an increase in the number of failures occurs with increase of the intensity of the received signal, i.e. the received signal differs from the original one, which cannot be due to incoherent radiation of the Sun. This behavior of the signal can be explained by the fact that it is subject of the resonance effect of the propagation medium, the nature of which has been intensively studied during recent years and is associated with the cascade of rescattering of electromagnetic waves on the states of Rydberg complexes $A^{* *} \mathrm{M}$ in the atmospheric $\mathrm{D}$ and $\mathrm{E}$ layers. This behavior of the signal can be explained by the effect of the propagation resonance medium, the nature of which has been intensively studied in recent years and is associated with the cascade of rescattering of electromagnetic waves on the states of Rydberg complexes $A^{* *} M$ in the lower atmosphere [26].

It was established in [33] that superfine UHF radiation is formed as a result of solar flares in the atmosphere. Intensity of this radiation in hundreds of times exceeds the typical levels of the microwave solar bursts. An analysis of the various possibilities for the generation of detected radiation indicates that the greatest contribution to the resulting spectrum pattern is done by transitions between the Rydberg states of the neutral components of a nonequilibrium two-temperature plasma excited by a stream of sunlight or a stream of electrons ejected from the ionosphere in collision with electrons. The layer of the atmosphere where such transitions predominantly occurs is located at a height of $80-110 \mathrm{~km}$ above the Earth's surface. During the satellite signal propagation through this layer, the multiple resonance scattering of photons occurs on the orbitally degenerate Rydberg complexes $A^{* *} M$ whose states are split due to the interaction with a neutral medium and between which the radiative transitions occur [19].

\section{Rydberg Molecules in Neutral Medium}

The Rydberg states are such highly excited states of atoms and molecules that are located near the ionization boundary and are characterized by presence of an infinite sequence of energy levels converging to the ionization threshold. Rydberg atoms and molecules have one excited weakly bound electron whose state is characterized by a level energy with a given principal quantum number $n$ and angular momentum 1 of the electron with respect to the ion core. The energies of levels with large angular momenta $l$ do not depend on the orbitally degenerate states. Thus, these states are statistically the most stable since electrons spend 
most of its time at large distances from the ion core.

The process leading to formation of the degenerate states with large angular momenta of the electron is called 1 mixing. In the upper atmosphere, it flows quickly and is irreversible. As a result, the quantum differences between the excited atoms and molecules disappear, and the radiation spectrum does not depend on their chemical composition [21]. The mixing process takes place in a sufficiently dense neutral gas medium with a density greater than $10^{12} \mathrm{~cm}^{-3}$ which corresponds to the heights $h \approx 110 \mathrm{~km}$. The criterion of its efficiency related directly to the density of the medium is the condition that at least one neutral molecule of oxygen or nitrogen exists in the volume of the electron cloud of the Rydberg molecule $\mathrm{A}^{* *}$ (with radius $2 n^{2} a_{0}$, where $a_{0}$ is the Bohr radius). The interaction between them leads to formation of the quasi-molecules $A^{\star *} M$, the potential energy surfaces (PES) of which are classified according to the angular momentum $L$ of the weakly bound electron with respect to the neutral $\mathrm{M}$ molecule. The shape of such a potential surface is determined by the characteristics of the elastic scattering of a slow electron on this molecule [34].

\section{Nonequilibrium Two-Temperature Plasma}

Ejection of ionosphere electrons passing through the E and D layers of the upper atmosphere occurs after intense flares on the Sun during periods of subsequent strong geomagnetic disturbances. Under these conditions the nonequilibrium two-temperature recombination plasma is formed where distribution of the Rydberg particles of the medium over the energy states occurs for the time of order of $10^{-10} \mathrm{~s}$ [21]. A spontaneous emission occurs much earlier than thermodynamic equilibrium is established. Since the concentration of free electrons $n_{e}$ is small in comparison with the concentration of atmospheric particles $\rho_{a}$, there is no noticeable change in temperature of the medium [34]; so, an influence of temperature deviations to atmospheric particles at the heights of $80-120$ $\mathrm{km}$ is small [35].

This is due to the fact that the high translational temperature of particles coming from the ionospheric F layer under entering a denser medium expended on the vibrational and rotational excitation of the atmospheric molecules [36]. Further relaxation of excitation is due to the processes of resonance transfer of internal energy, the transfer of which occurs due to subsequent collisions. As a result, separation of the electron temperature $T_{e}$ from the medium temperature $T_{a}$ occurs in the $\mathrm{D}$ and $\mathrm{E}$ layers, and a two-temperature recombination plasma with a temperature $T_{a} \ll T_{e}$ is established that agrees well with the direct measurements [37].

The neutral medium concentration for $\mathrm{E}$ and $\mathrm{D}$ atmosphere layers changes in the interval of $10^{12}<\rho_{a}<10^{16} \mathrm{~cm}^{-3}$. The concentration of highly excited particles formed here is determined by the temperature $T_{e}$ concentration $n_{e}$, and magnitude of the flux of free electrons, and also depends on the density of the medium. The states with the main quantum numbers at the range $n \approx 20-70$ 
are the most populated in the two-temperature recombination plasma. The concentration and temperature of the electrons here strongly depend on the levels of the magnetic storms (disturbances) within the limits of $10^{3} \leq n_{e} \leq 10^{6} \mathrm{~cm}^{-3}$ and $10^{3} \leq T_{e} \leq 3.5 \times 10^{3} \mathrm{~K}$, respectively [15]. An increase of the density in the lower part of the D layer should lead to an increase in the rate of the processes of shock and radiative quenching of the Rydberg states. Therefore, for $\rho_{a} \geq 10^{16} \mathrm{~cm}^{-3}$, their concentration will decrease sharply. The corresponding partial populations of the degenerate states of the Rydberg quasi-molecules are shown in Figure 4. They were calculated in [23] for the density of the medium and temperature corresponding to the heights at the lower part of the E layer and the upper part of the D layer. The curves depicted in Figure 4(a) correspond to the daytime when the concentration $n_{e}$ and temperature of free electrons $T_{e}$ under the influence of the Sun reaches $n_{e}=10^{6} \mathrm{~cm}^{-3}$ and $T_{e}=2000 \mathrm{~K}$. The population at the night time at $n_{e}=10^{4} \mathrm{~cm}^{-3}$ and $T_{e}=1000 \mathrm{~K}$ are presented in Figure 4 (b). It can be seen from Figure 4(a) \& Figure 4(b) that at night the level-by-level concentration of the Rydberg particles is less than in the daytime at least by three orders of magnitude.

\section{Incoherent High Frequency Radiation}

Preliminary calculations [23] have shown that the distribution of ultrahigh frequency (UHF) radiation lines dependent on $n$ and correspondingly on the values of $L=0-3$ for each molecule. They contain four series of $L \rightarrow L^{\prime}$ transition lines which with increase of the $L^{\prime}$ converge to the transition of $L \rightarrow n$ limit. The shift of the frequency limits relative to each other for nitrogen and oxygen molecules leads to significant inhomogeneity of the resulting radiation spectrum. The intensity of the incoherent UHF radiation is visibly reduced due to the difference between the scattering characteristics of the slow electrons for oxygen and nitrogen molecules (scattering lengths, quadrupole moments, and static polarizabilities) in three spectral frequency ranges belonging to the $1-100 \mathrm{GHz}$. The first one includes the frequency range of $1.17-1.71 \mathrm{GHz}$, the second-4.31 $6.09 \mathrm{GHz}$, and the third-7.27 - 57.1 GHz.

The dependences of the intensity of incoherent UHF radiation of the excited medium in the range of $0.8-1.8 \mathrm{GHz}$ for the noiseless and perturbed ionosphere are given in Figure 5(a) \& Figure 5(b) [15]. It can be seen that profile of the UHF radiation is a nonmonotonic function of the frequency $v$ and increases sharply near the right limit of the range. The relative intensities $W$ increase by about four orders of magnitude with increase of the electron concentration $n_{e}$ by two orders of magnitude. It is directly related to the effect of consistent reduction in the signal/noise ratio for the frequencies $L_{1}$ and $L_{2}$ of GPS signals during the solar flare power increase, since the position of the first range (1.17 $1.71 \mathrm{GHz}$ ) of the radiation intensity attenuation and the "transparency window" of the satellite signal propagation are practically coincided [23].

Note, that for sufficiently large values $(n>40)$ in the cross sections of the $I$ 


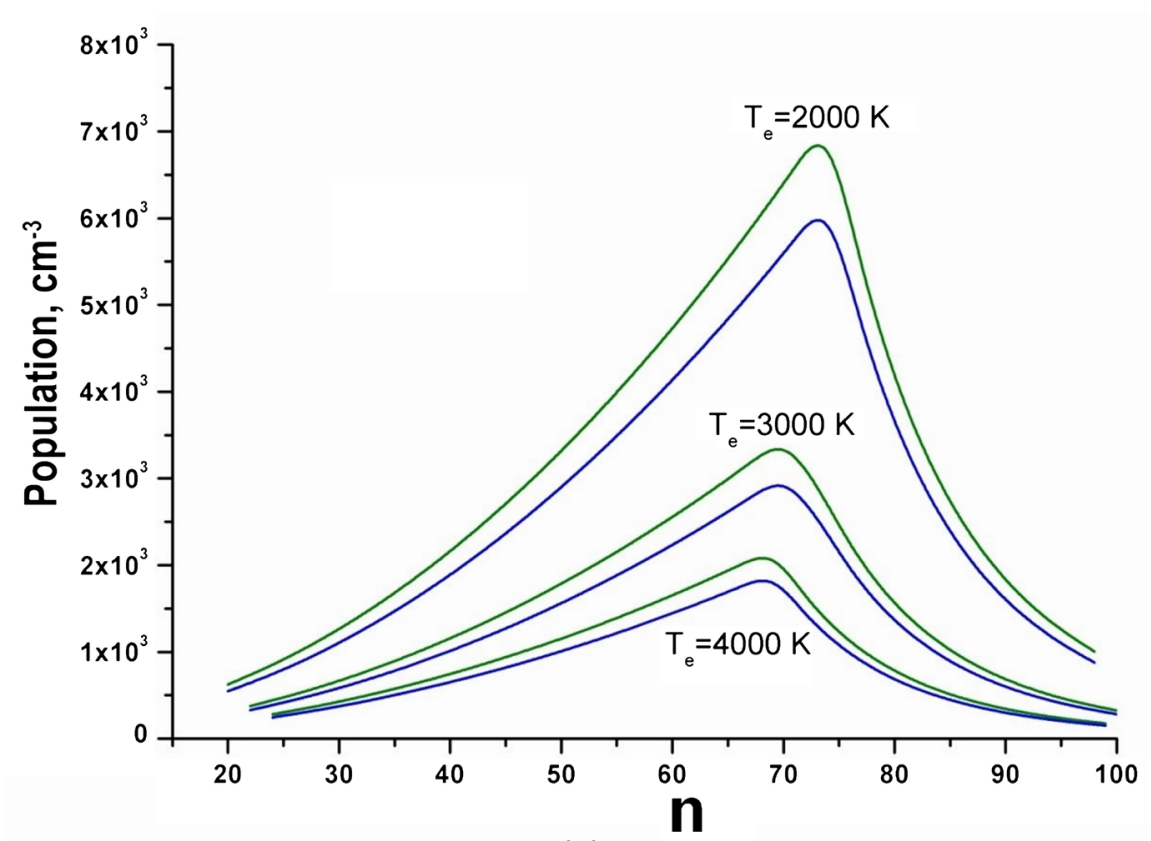

(a)

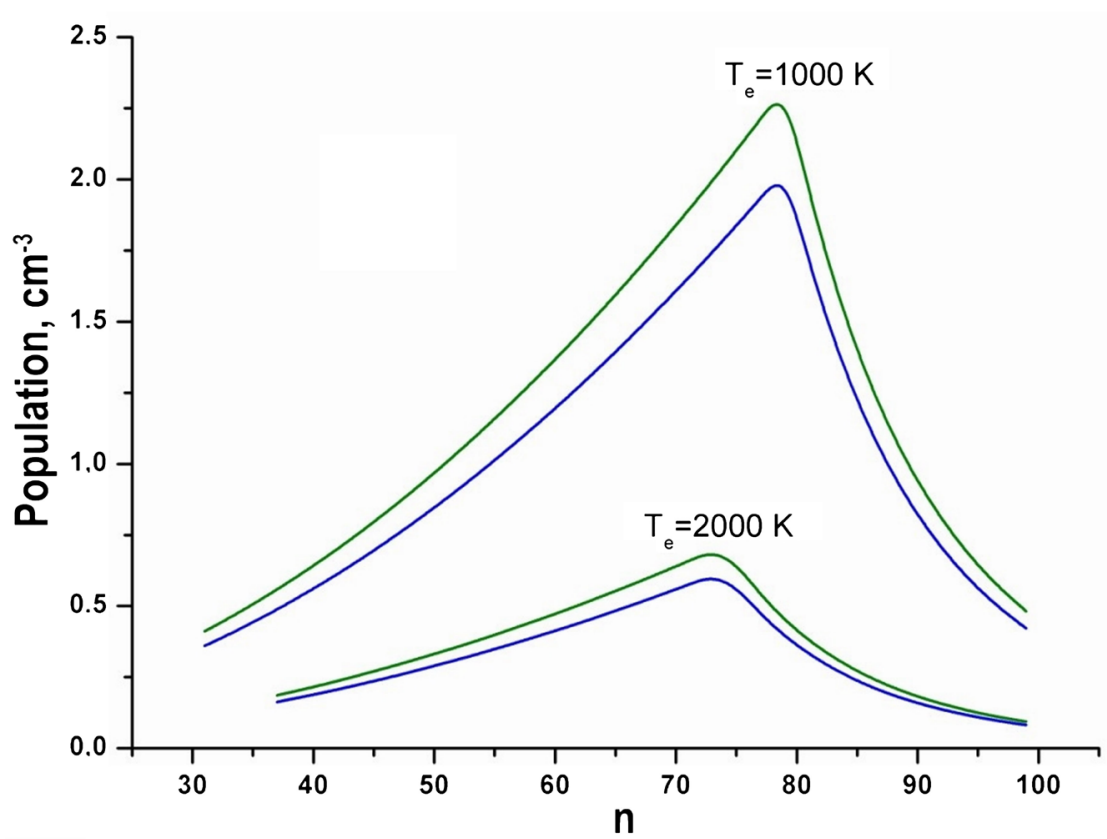

(b)

Figure 4. Dependencies of the populations of highly excited states of $A^{\star \star} N_{2}$ and $A^{\star \star} O_{2}$ quasi-molecules on the principal quantum number $n$ calculated at various electron temperatures $T_{e}$ and concentrations $n_{e}$ and medium density $\rho_{a}=10^{12} \mathrm{~cm}^{-3}$. Case (a) $n_{e}=10^{6} \mathrm{~cm}^{-3}$ corresponds to daily time; case (b) $n_{e}=10^{4} \mathrm{~cm}^{-3}$ corresponds to night.

mixing processes for the Rydberg molecules $\mathrm{N}_{2}^{* *}$ and $\mathrm{O}_{2}^{* *}$, a strong nonadiabatic coupling with rotation must be manifested [25] [26] [38]. This leads to formation of sharp dips at certain values $n_{\min }$ depending on the partial and total cross sections of $n$. The forms of dips are determined by the quantum properties of the colliding particles and their relative velocity. 


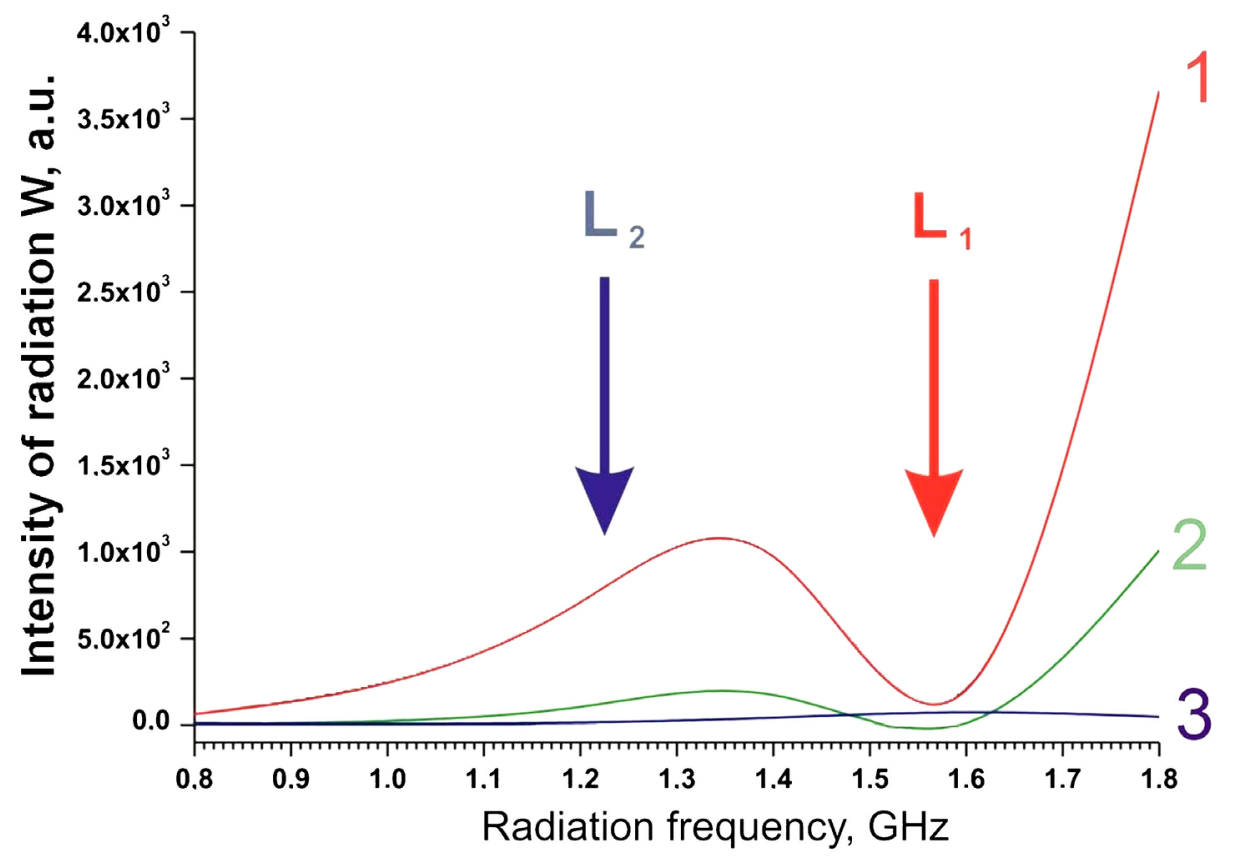

(a)

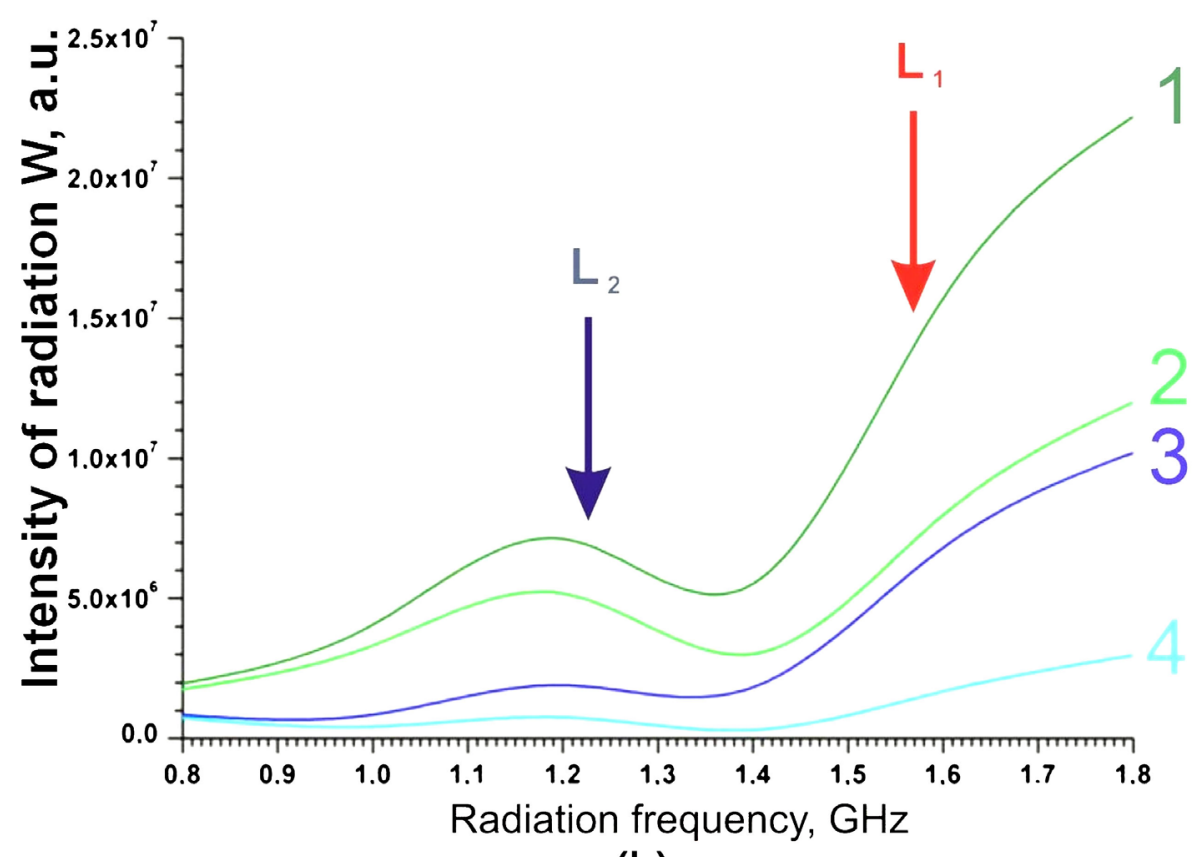

(b)

Figure 5. Dependences of the radiation intensities of highly excited quasi-molecules $A^{* *} N$ and $\mathrm{A}^{* *} \mathrm{O}$ on the frequency in the range $0.8-1.8 \mathrm{GHz}$ for various values of the density of the medium $\rho_{a}$ and the electron temperature $T_{e}$ for the electron concentrations equal $n_{e}=10^{3} \mathrm{~cm}^{-3}$ (Figure 5(a)) and $n_{e}=10^{5} \mathrm{~cm}^{-3}$ (Figure 5(b)), respectively. In Figure 5(a) curve (1) corresponds to the electron temperature $T_{e}=1000 \mathrm{~K}$ and the concentration of the medium $\rho_{a}=10^{12}-10^{13} \mathrm{~cm}^{-3}$; curve (2) $T_{e}=1000 \mathrm{~K}$ and $\rho_{a}=10^{14} \mathrm{~cm}^{-3}$; curve (3) $T_{e}=2000 \mathrm{~K}$ and $\rho_{a}=10^{12}-10^{13} \mathrm{~cm}^{-3}$. In Figure 5(b): curve (1) $T_{e}=2000 \mathrm{~K}$ and $\rho_{a}=10^{12}-10^{13} \mathrm{~cm}^{-3}$; curve (2) $T_{e}=2000 \mathrm{~K}$ and $\rho_{a}=10^{14} \mathrm{~cm}^{-3}$; curve (3) $T_{e}=3000 \mathrm{~K}$ and $\rho_{a}=10^{12}-10^{13} \mathrm{~cm}^{-3}$; curve (4) $T_{e}=3000 \mathrm{~K}$ and $\rho_{a}=10^{14} \mathrm{~cm}^{-3}$. 
Under the aforementioned conditions, rotational dips should arise at low electron $T_{e}$ temperatures. For example, at $T_{e} \leq 1000 \mathrm{~K}$, the position of the first minimum of the cross section is reached in the vicinity of the point $n_{\min }=44$ (whose half-width is $\Delta n= \pm 2$ ) [25]. With an increase of the electron temperature $\Delta T_{e} \geq 200 \mathrm{~K}$, the mean value

$$
\bar{n}=\frac{n_{\min }}{\sqrt{1+\left(\Delta T_{e} / T_{e}\right)}}
$$

decreases enough rapidly and the cross-section reaches the plateau. The picture is strongly different in the case of $l$ mixing the oxygen molecule due to a strong shift $\bar{n}$ toward smaller numbers $n$ where collisional dips are absent. This is due to the fact that the harpoon mechanism makes a significant contribution to the 1 mixing for $\mathrm{O}_{2}^{* *}+\mathrm{O}_{2}$ и $\mathrm{N}_{2}^{* *}+\mathrm{O}_{2}$ processes through the formation of intermediate resonant ion configurations $\left(\mathrm{O}_{2}^{+}, \mathrm{N}_{2}^{+}\right)+\mathrm{O}_{2}^{-}$.

This is an illustrative example when the individual spectroscopic properties of quasi-molecules composed the Rydberg molecules of nitrogen $\mathrm{N}_{2}^{* *}$ and oxygen $\mathrm{O}_{2}^{* *}$ in a neutral medium determine the observed features of the influence of solar activity on the failures of the GPS system. The selectivity of the effect at the frequencies $L_{1}$ and $L_{2}$ is due to the nonmonotonic character of the UHF spectrum which depends on the level of solar activity [24] [25] [26]. In the mentioned publications, the basement of the theory was developed and the "Rydberg" program was created. These developments were used for calculation of the frequency profiles of the power of the UHF radiation flux in the range of $0.8-10$ $\mathrm{GHz}$, which are essentially nonmonotonic ones.

\section{Long-Wave Infrared Radiation}

The incoherent UHF radiation of the Rydberg states during periods of geomagnetic disturbances is accompanied by intense long-wavelength infrared (IR) radiation (with a wavelength exceeding $15 \mu \mathrm{m}$ ) [39]. Analysis of the spectrum of this radiation allows reconstructing the parameters of a two-temperature plasma (electron concentration and temperature) for solving the inverse problem. This will enable, in turn, to connect the characteristic delay times of satellite signals at different carrier frequencies with the intensity of the power flux intensity of the incoherent UHF radiation $I_{\text {tot }}$ relying on the unique properties of the frequency profile of the radiation near two points: $1.4 \mathrm{GHz}$ and $5 \mathrm{GHz}$. These parameters were firstly calculated with the employment of the "Rydberg" software in [15] [24] [25] and presented in Figure 6 and Figure 7.

The frequency behavior presented in Figure 6 is called as a "waist point". The position of this point on the frequency axis does not depend on the temperature $T_{e}$ of the electrons, while the value of the power flux of radiation $I_{\text {tot }}$ along the vertical axis increases quadratically with the increasing electron concentration $n_{e}$.

Note that such unique properties of the radiation of the upper atmosphere were independently detected in studies for the remote passive location of the 


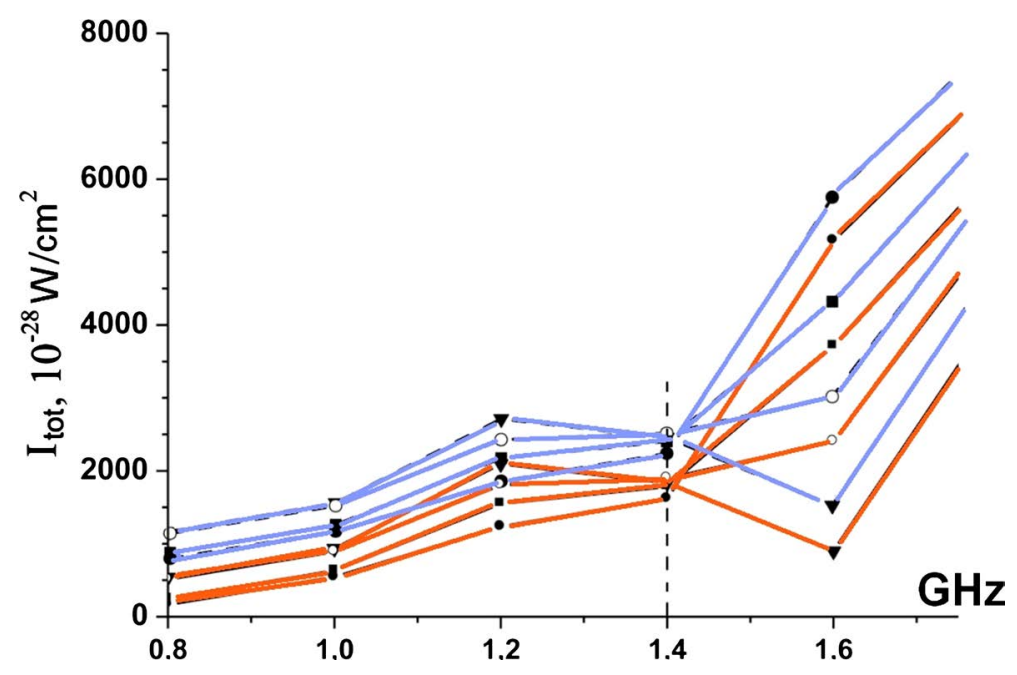

Figure 6. The dependence of the power flux of radiation $I_{\text {tot }}$ on the frequency $v$ for two electron concentration values: (1) $n_{e}=10^{4} \mathrm{~cm}^{-3}$ (red lines) and (2) $n_{e}=1.2 \times 10^{4} \mathrm{~cm}^{-3}$ (blue lines) and different electron temperatures $T_{e}: \boldsymbol{\nabla}-T_{e}=1000 \mathrm{~K}, \mathrm{O}-T_{e}=1200 \mathrm{~K}$, $-T_{e}=1500 \mathrm{~K}, \bullet-T_{e}=2000 \mathrm{~K}$.

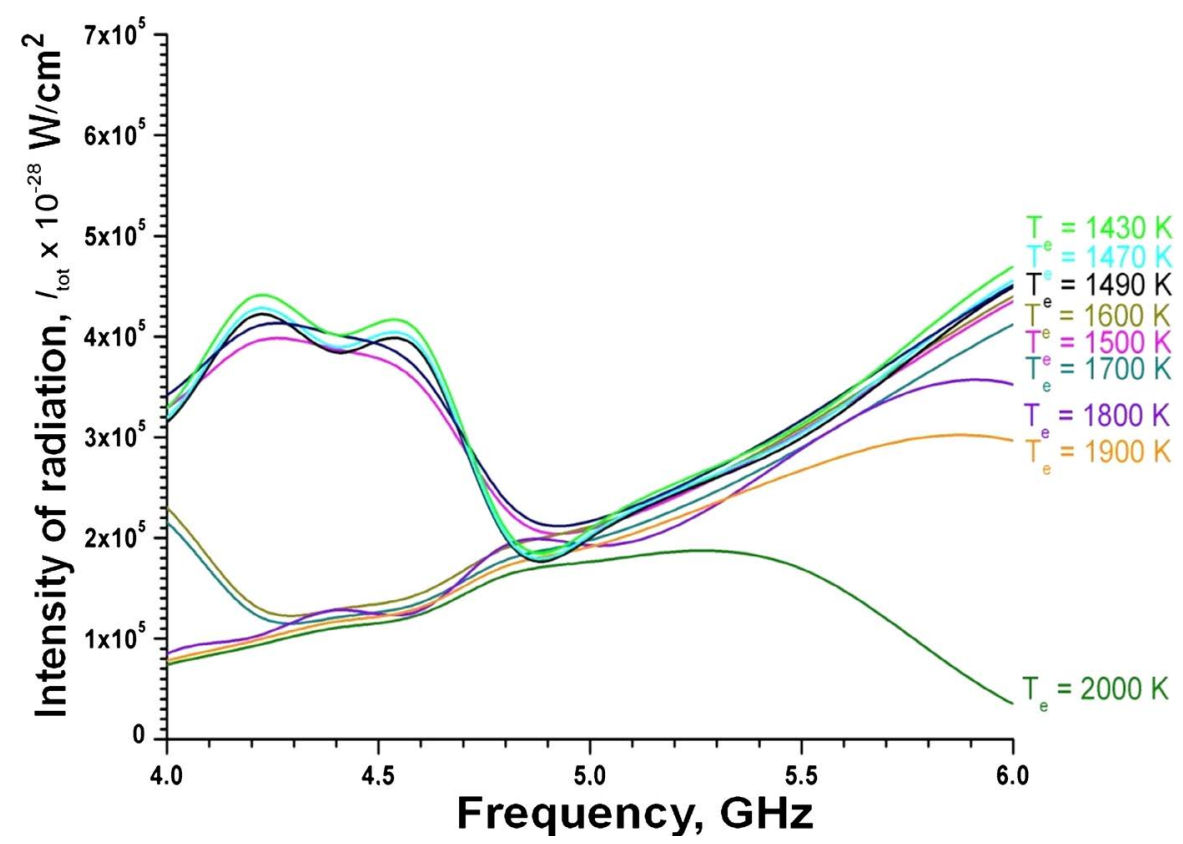

Figure 7. The dependence of the power flux of radiation $I_{\text {tot }}$ on the frequency $v$ for the electron concentration $n_{e}=10^{4} \mathrm{~cm}^{-3}$ and different temperatures $T_{e}$

Earth's surface [40].

\section{Discussion}

\subsection{Quantum Approach for Description of Satellite Signal Propagation}

The quantum theory of radiation, propagation and absorption of light originated in the beginning of the $20^{\text {th }}$ century and described in detail in various courses of 
general and theoretical physics (see, for example, [41]). In our problem, we will talk about the features of the passage of photons radiated by satellites through a resonance quantum medium in the $D$ and $E$ layers of the upper atmosphere of the Earth. Suppose that the transmitter signal is a certain group $N_{0}$ of photons is radiated at time $\tau_{0}$ in a certain order. The energy of a photon (here, and in the future, we will use the atomic system of units $\hbar=e=m_{e}=1$ ) is defined as $E_{0}=2 \pi v_{0}$, where $v_{0}$ is the carrier frequency of the transmitter which is assumed to be given. The total energy of the group will be $E\left(N_{0}\right)=2 \pi N_{0} v_{0}$, where $N_{0} \gg 1$. For simplicity, we write the initial radiation power of the group (without taking into account the details of the emission of photons) as $P\left(v_{0}\right)=2 \pi N_{0} v_{0} / \tau_{0}$. Then the power of the radio emission flux has the form

$$
I\left(v_{0}\right)=\frac{2 \pi N_{0} v_{0}}{\tau_{0} S},
$$

where $S$ is the flux area.

During a satellite signal propagates through the D and E layers of the atmosphere occurs scattering of photons moving in its electromagnetic field on the states of orbitally degenerate Rydberg complexes $A^{* *}$, which are split due to inelastic interaction with the neutral medium [15] [23] [24] [25] [26]. The symbol $A^{* *}$ denotes the highly excited states of atmospheric molecules $\mathrm{N}_{2}, \mathrm{O}_{2}, \mathrm{NO}$, and $M$, and includes the quadrupole diatomic molecules of nitrogen and oxygen. The total wave function of these states is superposition over the angular moments $l$ of an isolated electron (for $1<3$ ) and is characterized by the electron angular momentum $L$ with respect to the M molecule. The values of the level shifts $\Delta_{L}$ of a complex split off from the degenerate Coulomb levels by the interaction of the Rydberg molecule $A^{* *}$ with the neutral medium molecules $M$ depend on their main parameters (electron scattering length $\alpha$, polarizability $\beta$, and quadrupole moment $Q$ ) and appreciably (by an amount of order $n^{2}$, where $n$ is the principal quantum number) exceed the shifts $\Delta_{l}$ of isolated Rydberg levels (for $l \leq 3$ ), i.e.

$$
\Delta_{L} \sim \frac{a}{n^{4}} \gg \Delta_{l} .
$$

Under these conditions, an influence of the neutral medium reduces to an irreversible $l$ mixing process $\mathrm{A}^{* *}(n, l \leq 3)+\mathrm{M} \rightarrow \mathrm{A}^{* *}(n, l \geq 3)+\mathrm{M}$ with formation of the long-lived orbitally degenerate $A^{* *} M$ complexes which are populated, as was noted above, in a two-temperature plasma at the heights of $60-110 \mathrm{~km}$ above the Earth. At the lower boundary of this domain the process of effective quenching of the Rydberg complexes occurs [15] [23]. The upper boundary of this domain corresponds to the restriction on the density of the medium for the realization of $I$ mixing process [15]. The characteristic frequency of transitions $v_{0}=\Delta_{L} / 2 \pi$ in the complex $\mathrm{A}^{* *} \mathrm{M}$ (occurring without changing of the principal quantum number $n$ ) in this region is of the order of $1 \mathrm{GHz}\left(\right.$ or $\left.10^{-5} \mathrm{eV}\right)$.

The strength of the satellite signal field is determined by its power and, according to Equation (2), is proportional to the number of photons $N_{0}$. Since 
the wavelengths of the satellite GPS signal belong to the decimeter range and are much larger than the complex size, all the transitions can be considered in the dipole approximation and represent the electromagnetic interaction operator $\mathrm{w}$ in the form of $\mathbf{V}=-\mathbf{f D}$, where $\mathbf{f}$ is the quantity of the field strength operator determined by the source power, and $\mathbf{D}$ is the dipole moment operator of the complex $\mathrm{A}^{\star *} \mathrm{M}[30]$.

Since a satellite signal during the propagation through the $\mathrm{D}$ and $\mathrm{E}$ layers is scattered on the orbitally degenerate states of the Rydberg complexes $A^{* *} \mathrm{M}$ resolved by interaction with the neutral medium molecules [15] [22] [23], we associate the processes occurring here with their vibronic potential energy surfaces (PES).

\subsection{Types of Transition in Interaction of Photon with the Rydberg Complex}

The description of the orbitally degenerate states of Rydberg complexes $A^{* *} M$ requires, at the first stage, the introduction of diabatic vibronic potential energy surfaces (PES) $U_{n_{V} L}\left(\mathbf{R}, \xi_{\mathrm{M}}, u\right)$, where the vector $\mathbf{R}$ is the distance between the diatomic molecules $\mathrm{A}^{* *}$ and $\mathrm{M}, u$ is their relative velocity, and the vector $\xi_{\mathrm{M}}$ specifies the orientation of the molecule $\mathrm{M}$ in the coordinate system associated with the molecule $A^{* *}$. Under vibronic we mean the electron-vibrational states of the Rydberg complex with the principal quantum number $n_{\mathrm{V}}$ (depending on the vibrational quantum number $v$ of the molecular ion $A^{+}$) and the moment of the weakly bound electron $L$ relative to the molecule M. A regular method for constructing such PES is described in [34], and the wave functions should be constructed by analogy with the approach developed in [42]

The rotation of the molecule $\mathrm{A}^{* *}$ can be considered by classical way; we assume the direction of its axis to be fixed averaging over the orientations at the last stage of the calculation [43]. In addition, we should average here over the velocities $u$, confining ourselves to the Maxwellian distribution, since the main contribution is made by the domain of relative energy that is less or equal to the temperature $T_{a}$ of the neutral medium. Since the radiation processes in the problem formulation are assumed as quasistatic ones, the frequency of radiation (or absorption) transitions is determined by the relation

$$
v_{\mathrm{v}}\left(L_{i} \leftrightarrow L_{f}\right)=\frac{1}{2 \pi}\left|U_{n_{\mathrm{V}}, L_{i}}\left(\mathbf{R}, \xi_{\mathrm{M}}, u\right)-U_{n_{\mathrm{V}}, L_{f}}\left(\mathbf{R}, \xi_{\mathrm{M}}, u\right)\right| .
$$

Therefore, all subsequent averaging over orientations and relative velocities should be performed for a given transition frequency $v$.

To understand the physics of the phenomenon, it suffices to confine ourselves to three types of transitions. First type concerns to the intrinsic spontaneous ultrahigh frequency (UHF) radiation of a neutral quantum medium, which is incoherent and propagates in an arbitrary direction. The dissociation of the Rydberg complexes $A^{* *} M$ does not take into account in this case since the distribution of populations of the Rydberg complexes with respect to binding energy 
$E_{n}$ in a non-equilibrium plasma is assumed to be stationary and is maintained by a constant flux of free electrons. The distribution of the populations of levels located above some binding energy $E_{*}$ is practically equilibrium with an electron temperature close to the temperature of free electrons $T_{e}$ [23]. At the high binding energies $E_{n} \sim E_{*}$ equilibrium, distribution is strongly perturbed over the $\Delta E \ll E_{*}$ energy interval. This interval is called as a "sink neck" or a "narrow recombination flux place" [44]. Above the sink neck, where $E_{n}<E_{*}$, the collision transitions between the bound states and continuum is predominated. Below the neck, the radiative transitions predominate, that result in the equilibrium population of the low-lying states with a $T_{a}$ temperature of medium molecules. However, as was indicated above, the electron temperature $T_{e}$ here is much higher than the temperature of the ions $T_{i}$ and the neutral medium $T_{a} ; T_{i}$ and $T_{a}$ practically coincide in the $\mathrm{D}$ and $\mathrm{E}$ layers.

Second type is a direct transition $N_{0} \rightarrow N_{0}+1$ to the underlying state of the complex and corresponds to stimulated emission at the carrier frequency $v_{0}$ [45]. It preserves the polarization of the photons of the satellite signal and can be considered without taking into account a predissociation. Spontaneous emission of photon by a neutral resonance medium propagates in an arbitrary direction, while the stimulated emission retains the direction of the initial radiation incident at the Rydberg complex. Since the polarization of the stimulated emission coincides with the polarization of the satellite GPS signal, this leads to the creation of additional photons and the experimentally observed increase in the power of the received GPS signal. This leads to creation of additional photons and the experimentally observed power of the received GPS signal is increased. This effect was first discovered in Afraimovich's [13] unique experience, which was held at the Cornell University (USA) and has not been explained to the present. Direct measurements have shown that the power of the obtained receiver's radiation at the frequency $v_{0}$ increases about three-fold compared to the transmitter power at satellite and is accompanied by signal failures at the receiver (see Figure 2).

Third type of transitions corresponds to the resonance scattering of photon on the complex $A^{* *} \mathrm{M}$ which proceeds through three successive stages. The first one takes place during times of $10^{-15}-10^{-14} \mathrm{~s}$ and corresponds to the stimulated absorption of photon with $L \rightarrow L_{\text {int }}$ transition (for $\Delta n=0$ ) into the intermediate overlying $n_{\mathrm{V}}, L_{\text {int }}$ vibronic state of the complex.

These times (depending on the magnitude of $n$ ) correspond to the duration of the photon passage through the electron cloud of the Rydberg complex. The next stage is related to the quantum dynamics of the $A^{* *} M$ complex itself, where along with the discrete states, the energy spectrum of the composite system includes the virtual autoionization states of the $A^{* *}$ molecule [46]. This is realized when the relative energy of the diatomic molecules $A^{* *}$ and $M$ in the complex $E_{k} \geq T_{a}$ is greater than the binding energy of the electron, i.e. by condition $n_{0} \geq 1 / \sqrt{2 T_{a}} \geq 30$. The characteristic values of $n_{0}$, populated in a non-equilibrium 
plasma, occur at the altitudes of $80-110 \mathrm{~km}$ above the Earth, in the interval of 20 -70 [23].

The process of the resonant scattering of photon completes at the same time of order of $10^{-15}-10^{-14} \mathrm{~s}$ by the forced transition to the final state $N_{0}-1 \rightarrow N_{0}$. Note that the resonance scattering of photons on the orbitally degenerated Rydberg complexes includes, as shown in Figure 8, a lot of intermediate states, and therefore it fundamentally differs from the traditional scheme which not contains a time delay [41]. We also note that in the problem of the resonance scattering of Rydberg electron, the presence of predissocational channels should be taken into account when considering independently the dissociation of complexes for various fragments of a neutral medium [15] [23]. Thus, the theory of propagation and distortion of satellite signals should include simultaneously the contributions of all three aforementioned types of the radiation processes. Besides this, here the improved Kalman filtering with time delay between the useful signal and noise and wideband noise effect [7] could be applied.

\subsection{Theory of Resonance Scattering of Photon by Rydberg Complex}

Let us discuss the development of a general scheme of the formal theory of resonance scattering of photon. The dipole matrix element of the transition from the initial $n_{V} L$ state to the intermediate overlying $n_{V} L_{\text {int }}$ state of the Rydberg

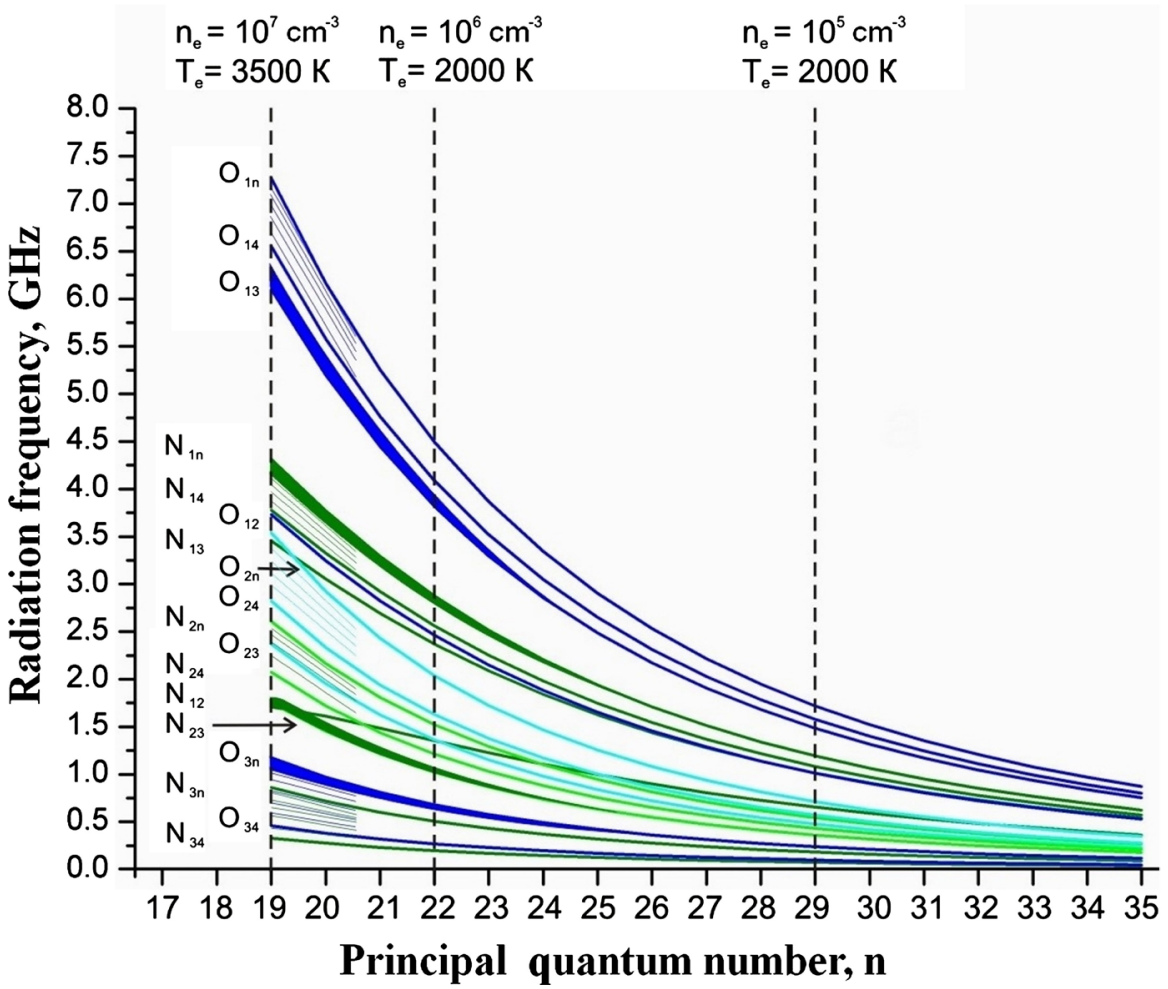

Figure 8. Dependence of absorption (emission) lines of Rydberg complexes $A^{\star \star} N_{2}$ and $\mathrm{A}^{* *} \mathrm{O}_{2}$ on the principal quantum number $n$. The bold lines denote the boundaries of the ranges of attenuation of the intensity of absorption (radiation). 
complex $\mathrm{A}^{* *} \mathrm{M}$ describing the first act of the photon absorption has the form

$$
\mathbf{D}_{n_{\mathrm{V}}}\left(L \rightarrow L_{\text {int }}\right)=\left\langle\Psi_{n_{\mathrm{V}} L}\left(\mathbf{r}, \mathbf{R}, \xi_{\mathrm{M}}\right)|\mathbf{r}| \Psi_{n_{\mathrm{V}} L_{\text {int }}}\left(\mathbf{r}, \mathbf{R}, \xi_{\mathrm{M}}, u\right)\right\rangle .
$$

Here $\Psi_{n_{\mathrm{V}} L}$ is the total wave function of the initial vibronic complex state (for the fixed values of vectors $\mathbf{R}$ and $\left.\xi_{\mathrm{M}}\right), \Psi_{n_{\mathrm{v}} L_{\text {int }}}$ is the total wave function of the intermediate autoionization complex state which describes the dynamics of the behavior of the Rydberg electron moving in the two-center force field of the quantum system "Rydberg molecule A ** + neutral molecule of the medium M".

\subsubsection{Vibronic Wave Function of the Initial State of a Complex}

The vibronic wave functions $\Psi_{n_{\mathrm{V}} L}$ of two-center system satisfy to the exact relation for the level shift operator $\tau$ in the formal theory of scattering [42]

$$
\boldsymbol{\tau}\left|\Phi_{n_{\mathrm{V}} L}\right\rangle=\mathbf{V}_{e^{-} M}\left|\Psi_{n_{\mathrm{V}} L}\right\rangle \text {. }
$$

Here $\mathbf{V}_{e^{-} M}$ is the operator of $e^{-}-M$ interaction, $\Phi_{n_{\mathrm{V}} L}$ is the basis wave function of a non-interacting system in the $L$-representation normalized to unity [43]. The operator $\tau$ satisfies to the integral Lippman-Schwinger equation

$$
\boldsymbol{\tau}=\mathbf{V}_{e^{-} M} \mathbf{G}_{A^{* *} M}(E) \boldsymbol{\tau},
$$

where the Green's operator $\mathbf{G}_{A^{* *} M}$ of the Rydberg molecule is determined as usually in accordance with [43]. It follows from the integral Equation (7) that the wave functions of the Rydberg complex $\mathrm{A}^{* *} \mathrm{M}$ can be represented in the form

$$
\left|\Psi_{n_{\mathrm{V}} L}\right\rangle=\mathbf{V}_{e^{-} M}^{-1} \boldsymbol{\tau}\left|\Phi_{n_{\mathrm{V}} L}\right\rangle=\mathbf{G}_{A^{* *} M} \boldsymbol{\tau}\left|\Phi_{n_{\mathrm{V}} L}\right\rangle
$$

and it is expressed in the terms of the corresponding matrix elements of the $\mathrm{D}$ level of the shift operator $\tau$.

\subsubsection{Wave Function of the Intermediate Autoionization State}

The total vibronic wave function $\Psi_{n_{\mathrm{V}} L_{\text {int }}}(u)$ of the intermediate autoionization

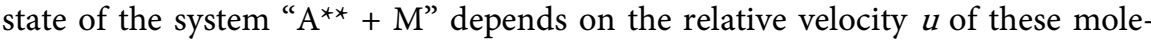
cules and by analogy with Equation (6) is also defined as

$$
\left|\Psi_{n_{\mathrm{V}} L}(u)\right\rangle=\mathbf{V}_{e^{-} M}^{-1} \boldsymbol{\tau}\left|\Phi_{n_{\mathrm{V}} L}(u)\right\rangle=\mathbf{G}_{A^{* *} M}(E) \boldsymbol{\tau}\left|\Phi_{n_{\mathrm{V}} L}(u)\right\rangle .
$$

Here $E=-\frac{1}{2 n^{2}}+E_{k}$ is the total energy of the system counting from the molecular ion $A^{+}$ground state, $E_{k}$ is the kinetic energy equal to $E_{k}=\frac{M_{c} u^{2}}{2}, M$ is the reduced mass of the particles $A^{* *}$ and $M$. The difference between Equations ((9) and (7)) is that the operator $\mathbf{V}_{e^{-} M}(u)$ describes the $e^{-}-M$ interaction of the electron with the molecule $M$, which moves with a given velocity $u$ in the coordinate system is associated with Rydberg molecule $\mathrm{A}^{* *}$. In this case, the wave function $\Psi_{n_{\mathrm{V}} L}(u)$ is written in the $L$-representation and normalized by the $\partial$-function of the energy $Q$ of relative motion. In determining the operator $\mathbf{V}_{e^{-} M}(u)$ it suffices to regard the Rydberg electron as free one and to neglect the dependence from $u$. Such a representation is valid if the wavelength $\lambda$ of electron changes is smaller on dimensions of the effective domain $\alpha$ of interaction 
with the neutral particle $M$, i.e. under condition [34]

$$
\alpha \frac{\mathrm{d} \lambda}{\mathrm{d} r} \ll \lambda \text { or } \frac{\alpha}{\left(p_{e} R\right)^{2}} \ll 1
$$

( $p_{e}$ is the classical electron momentum in a Coulomb field).

The main role in Equation (9) plays the Green's operator $\mathbf{G}_{A^{* * *} M}(E)$ which by definition is a convolution [35]

$$
\mathbf{G}_{A^{* *} M}\left(\mathbf{R}, \mathbf{R}^{\prime}, E\right)=\frac{1}{2 \pi^{2}} \int_{0}^{k_{\max }} \mathbf{G}_{A^{* *}}\left(E-E_{k}\right) \frac{\sin \left[\mathbf{k}\left(\mathbf{R}-\mathbf{R}^{\prime}\right)\right]}{\left|\mathbf{R}-\mathbf{R}^{\prime}\right|} k \mathrm{~d} k .
$$

Here $\mathbf{G}_{A^{* *}}$ is the Green's operator of the isolated Rydberg molecule $\mathrm{A}^{* *}$, the quantities $E_{k}$ and $k_{\max }$ are respectively equal to $E_{k}=k^{2} / 2 M_{C}$, $k_{\max }=\sqrt{2 M_{c}\left(E+\frac{1}{R}\right)}$. In the case of positive energy $E>E_{k}$, the operator $\mathbf{G}_{A^{* *}}$ in Equation (11) describes the motion of electron in the continuous spectrum. This region of the momentum variation $k$ of the colliding particles includes virtual transitions to the ionization continuum and transitions between its states. This operator satisfies to the reduced Dyson equation [43]

$$
\mathbf{G}_{A^{* *}}(E)=\mathbf{G}^{(C)}(E)+\mathbf{G}^{(C)}(E) \mathbf{T}^{(C)}(E) \mathbf{G}^{(C)}(E),
$$

where $\mathbf{G}^{(C)}$ is the Coulomb Green operator, and $\mathbf{T}^{(C)}$ is the collision matrix of an electron with the Rydberg molecule $\mathrm{A}^{\star *}$.

The poles of the Coulomb Green function here are strictly compensated and the eigenvalue spectrum is determined by the $\mathbf{T}^{(C)}$-matrix poles, which satisfies to the system of integral equations [42]

$$
\left\{\begin{array}{l}
\mathbf{T}^{(C)}=\mathbf{t}+\mathbf{t} \sum_{q}|q\rangle\langle q| \cot \left[\pi v_{q}(\varepsilon)\right] \mathbf{T}^{(C)} \\
\mathbf{t}=\mathbf{V}_{e^{-} A^{+}}+\mathbf{V}_{e^{-} A^{+}} \mathbf{G}_{0} \mathbf{t}
\end{array} .\right.
$$

The $\mathbf{t}$ operator in Equation (13) is the matrix of reactions which describes the electrostatic interaction of electron with ion core $\mathrm{A}^{+}$. The wave functions $|q\rangle=|l m v\rangle$ are the Coulomb basis functions, $l$ and $m$ are the angular momentum of the electron and its projection on the axis of the molecule $\mathrm{A}^{* *}$. The function $v_{q}(\varepsilon)$ is defined as $v_{q}(\varepsilon)=(-2 \varepsilon)^{-1 / 2}$. In going over to the continuous spectrum, the function $\cot [\pi v(\varepsilon)]=-i$.

Since for a given $m$ the states with different $l$ are mixed, the total normalized wave function of the orbital degenerate state of the molecule $A^{* *}$ is defined as the superposition

$$
\psi_{n l m}^{(L)}(\mathbf{r}, R)=\sum_{l \geq l^{*}}^{n-1} A_{n l m}(R) \Phi_{n l m}^{(L)}(\mathbf{r}, R),
$$

where $l^{*} \geq 3$, and the coefficients $A_{n l m}$ are equal to

$$
A_{n l m}(R)=\frac{\Phi_{n l m}^{(L)}(\mathbf{R}, R)}{\sum_{l \geq l^{*}}^{n-1}\left|\Phi_{n l m}^{(L)}(\mathbf{R}, R)\right|^{2}} .
$$


In this case, the partial wave functions $\Phi_{n l m}^{(L)}$ are normalized to unity for each value of $R$. Thus, in Eq. (12) the resonance terms appear in the scattering of electron by the molecule $A^{* *}$ and a finite lifetime of the electron in the ionization continuum arises respectively.As a result, the wave function $\Psi_{n_{\mathrm{V}} L_{\text {int }}}(u)$ of the intermediate autoionizing state of the complex $A^{\star *} M$ is expressed in terms of the characteristics of the resonance scattering of electron by the molecule $\mathrm{A}^{* *}$. Therefore, this is the reason for the photon delay in the intermediate state of the $\mathrm{A}^{* *} \mathrm{M}$ complex due to interaction with the neutral medium.

\subsection{Propagation and Distortion of a Radio-Signal}

For a qualitative explanation of the features of GPS signal distortion during the propagation through the $80-110 \mathrm{~km}$ layer, it suffices to confine ourselves to the case of the resonance (Rayleigh) elastic scattering (when the quantum state of the Rydberg complex $\mathrm{A}^{\star \star} \mathrm{M}$ does not change) and to analyze the resonant structure of the transition oscillator strength $F_{n_{\mathrm{V}}}\left(L \rightarrow L^{\prime}\right)$. The problem of the radiative transition to the ionization continuum of the Rydberg molecule (bound-free transitions) was considered in [47] [48], where the total wave functions of the molecule are expressed in terms of the collision $\mathrm{T}$ - matrix elements describing the slow electron scattering on the ion core in the Coulomb potential and the force field of the $M$ molecule [43]. As a result, the dipole matrix element $n_{\mathrm{V}} L \rightarrow n_{\mathrm{V}} L^{\prime}$ transition becomes superposition of the direct and two-stage (resonant) processes, and the strength of the transition oscillator acquires a typical form of the contour Fano. For predissociation states (lying in ionization continuum) such a situation arises with the participation of discrete vibronic levels of the vibrational excited states of the A+ ion (located near the corresponding levels of the series $(v=0)$, when the connection between the Rydberg configuration and the dissociative continuum increases substantially due to the decrease of the $I$ parameter and principal quantum number $n_{\mathrm{V}}$, since the total wave function includes the superposition of all possible vibronic states with a given energy. As a result, the strength of the transition oscillator can be represented in the form [48]

$$
F_{n_{\mathrm{V}}}\left(L \rightarrow L^{\prime}\right)=F_{n_{\mathrm{V}}}^{(0)}\left(L \rightarrow L^{\prime}\right)\left[\frac{\left(q_{n_{\mathrm{V}}}+\delta_{n_{\mathrm{V}}}\right)^{2}}{\left(1+\delta_{n_{\mathrm{V}}}^{2}\right)}\right]^{4},
$$

where $F_{n_{\mathrm{V}}}^{(0)}$ is the strength of the direct transition oscillator, $q_{n_{\mathrm{V}}}$ is the profile index, $\partial_{n_{\mathrm{V}}}=4 \pi \Delta v / \Gamma_{n_{\mathrm{V}}}$ is the shift of the carrier frequency, and $\Gamma_{n_{\mathrm{V}}}$ is the width of the resonant level.

It can be seen that the frequency shift of the scattered photon $\partial v_{0}$ and the delay time $\partial \tau$ in the intermediate state (due to the broadening of the autoionization discrete levels) are completely determined by the strong nonadiabatic coupling of the electron and nuclear motions in the intermediate complex $\mathrm{A}^{* *} \mathrm{M}$ and by the configuration interaction $\mathbf{V}^{\mathrm{CI}}$ with a dissociative continuum. The corresponding complex dipole moment of $L \rightarrow L^{\prime}$ transitions is formed by an 
entire set of principal quantum numbers $n_{V}$ (see Figure 8). The selection rule here has $\mathrm{A}$ form of $\Delta L= \pm k$ (where $k$ is an integer), which arises due to the lack of the central symmetry for the Rydberg molecules in the system $A^{\star \star}+M$ [23] [26].

Preliminary estimates of the single-photon resonant scattering give the following values: for the frequency shift $\partial v_{0} \sim 10^{-2} \mathrm{GHz}$ and the time delay $\partial \tau \sim 10^{-10} \mathrm{~s}$. Consequently, the formation of observable values of delay times during the propagation of a satellite signal through the atmospheric layer of 80 $110 \mathrm{~km}$ should be realized as a result of no less than $10^{6}$ scattering events of the photon group $N_{0}$ that has a good agreement with the calculations [25], since the total number of the Rydberg complexes in a column with the area on the receiver antenna under normal conditions for $10 \mathrm{~cm}^{2}$ is $\sim 10^{7}-10^{8}$. Considering that the velocity propagation of a signal is much higher than the characteristic velocities of molecules inside the complexes, it suffices to confine oneself to their quasistatic description. Then, taking into account a normal distribution for the Brownian motion and considering that the probability of one event of a resonance scattering of photon is $w \sim 10^{-3}$ for $10^{6}$ interactions, we obtain $\Delta \tau \sim 10^{-7} \mathrm{~s}$, i.e. about 100 nanoseconds.

Thus, the Rydberg complexes $A^{* *} M$ serve peculiar traps for the scattered photons. It should be pointed out that a similar phenomenon was considered earlier for metallic particle [49].

\section{Conclusion}

It should be noted that up to the present day, empirical Equation (1), which was obtained as a result of statistical processing of the received signals under normal geomagnetic conditions, is used in the GNSS positioning satellite systems for calculating the delay time of a signal. However, magnetic disturbances (and especially storms) significantly change the concentration and flow of electrons in the Earth's atmosphere. Various attempts to mathematically-physically (and chemically) describe such situation within the framework of classical electrodynamics cannot in principle lead to the delay since it is well known (and experimentally confirmed) that the refractive index in the ionosphere (including plasma inhomogeneities) differs from unity only in the fourth decimal place [36]. It is the resonant multiple interaction of a signal with a quantum medium that determines the delay process: this description is firstly proposed and formulated by the authors of this paper. The development of a general theory of signal propagation and delay should be based on an integral version of the theory of a multichannel quantum defect (MQD) and is divided into three stages. The first assumes the construction of a complete adiabatic wave function of the initial state of the Rydberg complex, including the vibronic states and the exchange interaction (for the case where the Rydberg molecule and the neutral molecule of the medium are coincided). To solve this problem, we proposed to use the method developed in [42] for a simple system "Rydberg atom + atom with a filled elec- 
tron shell" and substantially refine it. Secondly, it is necessary to develop a regular method for constructing the complete wave function of the complex taking into account the dynamics of its fragments when the kinetic energy of their relative motion is included to the general consideration [46], which was also not earlier discussed and is done for the first time. This will allow us at the third stage to determine the corresponding dipole matrix elements of the resonant scattering to introduce into the theory the power of the radiation obtained at receiver and the total delay time of the signal as a result of the rescattering cascade along the propagation path of the signal. At the same time, it is necessary to develop a numerical program and calculate the total delay time of the satellite signal, depending on the main parameters of the non-equilibrium plasma (concentration, flux, and electron temperature) for various geomagnetic conditions occurring in the ionosphere. In conclusion, we note that a consistent quantum theory of the propagation of satellite signals for a more complete imagination of the phenomenon must also take into account the Raman scattering of photons and the possibility of decay of $\mathrm{A}^{\star *} \mathrm{M}$ complexes, which have not been discussed yet. This will allow to calculated reliable values of the carrier frequency shift and the delay time of the radio waves incoming to the Earth.

\section{Acknowledgements}

This work was supported by the Russian Foundation of Basic Investigations (Grant 16-05-00052).

\section{Conflicts of Interest}

The authors declare no conflicts of interest regarding the publication of this paper.

\section{References}

[1] Zumberg, J.F., Heftin, M.B., Jeffersonet, D.C., et al. (1997) Precise Point Positioning for the Efficient and Robust Analysis of GPS Data from Large Networks. Journal of Geophysical Research: Solid Earth, 102, 5005-5017. https://doi.org/10.1029/96JB03860

[2] Wang, W.J., Hsu, T.M. and Wu, T.S. (2017) The Improved Pure Pursuit Algorithm for Autonomous Driving Advanced System. IEEE 10th International Workshop on Computational Intelligence and Applications (IWCIA), Hiroshima, 11-12 November 2017, 33-38. https://doi.org/10.1109/IWCIA.2017.8203557

[3] Eppelbaum, L.V. (2013) Non-Stochastic Long-Term Prediction Model for US Tornado Level. Natural Hazards, 69, 2269-2278. https://doi.org/10.1007/s11069-013-0787-7

[4] Kaplan, E.D. and Hegarty C.J. (2006) Understanding GPS Principles and Applications. 2nd Edition, Artech House, Norwood.

[5] Finkelstein, M., Price, C. and Eppelbaum, L. (2012) Is the Geodynamic Process in Preparation of Strong Earthquakes Reflected in the Geomagnetic Field? Journal of Geophysics and Engineering, 9, 585-594. https://doi.org/10.1088/1742-2132/9/5/585

[6] Eppelbaum, L.V. (2011) Study of Magnetic Anomalies over Archaeological Targets 
in Urban Conditions. Physics and Chemistry of the Earth, 36, 1318-1330. https://doi.org/10.1016/j.pce.2011.02.005

[7] Eppelbaum, L.V. and Mishne, A.R. (2011) Unmanned Airborne Magnetic and VLF investigations: Effective Geophysical Methodology of the Near Future. Positioning, 2, 112-133. https://doi.org/10.4236/pos.2011.23012

[8] Eppelbaum, L.V., Katz, Y.I. and Ben-Avraham, Z. (2012) Israel-Petroleum Geology and Prospective Provinces. AAPG European Newsletter, 4, 4-9.

[9] Rampinini, E., Alberti, G., Fiorenza, M., Riggio, M., Sassi, R., Borges, T.O. and Coutts, A.J. (2004) Accuracy of GPS Devices for Measuring High-Intensity Running in Field-Based Team Sports. International Journal of Sports Medicine, 36, 49-53.

[10] Eppelbaum, L.V. and Katz, Y.I. (2015) Paleomagnetic Mapping in Various Areas of the Easternmost Mediterranean Based on an Integrated Geological-Geophysical Analysis. In: Eppelbaum, L., Ed., New Developments in Paleomagnetism Research, Ser. Earth Sciences in the 21 st Century, Nova Science Publisher, New York, 15-52.

[11] Eppelbaum, L.V. (2016) Remote Operated Vehicles Geophysical Surveys in Air Land (Underground) and Submarine Archaeology: General Peculiarities of Processing and Interpretation. Translation of the 12 th EUG Meeting. Geophysical Research Abstracts, Vol. 18, Vienna, 17-22 April 2016, 1-7.

[12] Cerruti, A.P., Kintner Jr., P.M., Gary, D.E., et al. (2008) Effect of Intense December 2006 Solar Radio Bursts on GPS Receivers. Space Weather, 6, 1-10.

https://doi.org/10.1029/2007SW000375

[13] http://gps.ece.cornell.edu/realtime.php

[14] Afraimovich, E.L., Astafieva, E.I., Berngardt, O.I., et al. (2004) Mid-Latitude Amplitude Scintillation of GPS Signals and GPS Performance Slips at the Auroral Oval Boundary. Radiophysics and Quantum Electronics, 47, 453-468. https://doi.org/10.1023/B:RAQE.0000047237.67771.bc

[15] Golubkov, G.V., Golubkov, M.G. and Manzhelii, M.I. (2012) Microwave and IR Radiation of the Upper Atmosphere during Periods of Enhanced Solar Activity. Doklady Physics, 57, 461-464. https://doi.org/10.1134/S102833581212004X

[16] Montenbruck, O. and Markgraf, M. (2001) Orion GPS Tracking System. Flight Report Max4-DLR-RP-0001.

[17] Markgraf, M. (2005) Phoenix GPS Tracking System. Flight Report VSB 30-DLRRP-0001.

[18] Hauschild, A., Markgraf, M. and Montenbruck, O. (2014) The Navigation and Occultation Experiment GPS Receiver on Board a LEO Satellite. Inside GNSS, 9, 49-57.

[19] Golubkov, G.V., Manzhelii, M.I. and Karpov, I.V. (2013) Ultrahigh Frequency Additional Background Radiation of the Lower Ionosphere during Strong Geomagnetic Disturbances. Russian Jour. of Physical Chemistry B, 7, 641-651. https://doi.org/10.1134/S1990793113050175

[20] http://gps.ece.cornell.edu/x6flare.php

[21] Golubkov, G.V., Manzhelii, M.I. and Karpov, I.V. (2011) Chemical Physics of the Upper Atmosphere. Russian Journal of Physical Chemistry B, 5, 406-411. https://doi.org/10.1134/S1990793111030055

[22] Golubkov, G.V. (2011) Influence of the Medium on the Electromagnetic Radiation Spectrum of Highly Excited Atoms and Molecules. Russian Journal of Physical Chemistry B, 5, 925-930. https://doi.org/10.1134/S1990793111060108

[23] Golubkov, G.V., Golubkov, M.G. and Manzhelii, M.I. (2012) Microwave Radiation in the Upper Atmosphere of the Earth during Strong Geomagnetic Disturbances. 
Russian Journal of Physical Chemistry B, 6, 112-127. https://doi.org/10.1134/S1990793112010186

[24] Golubkov, G.V., Golubkov, M.G. and Manzhelii, M.I. (2013) Additional Background Radiation of Atmosphere D-Layer at 0.8 to $6.0 \mathrm{GHz}$. Doklady Physics, 58, 424-427. https://doi.org/10.1134/S1028335813100066

[25] Golubkov, G.V., Golubkov, M.G. and Manzhelii, M.I. (2014) Rydberg States in the Atmosphere D Layer and GPS System Positioning Errors. Russian Journal of Physical Chemistry B, 8, 103-115. https://doi.org/10.1134/S1990793114010126

[26] Golubkov, G.V., Golubkov, M.G., Manzhelii, M.I. and Karpov, I.V. (2014) Optical Quantum Properties of GPS Signal Propagation Medium-D layer. In: Bychkov, V.L., Golubkov, G.V. and Nikitin, A.I., Eds., The Atmosphere and Ionosphere: Elementary Processes, Monitoring, and Ball Lighting, Springer, New York, 1-68. https://doi.org/10.1007/978-3-319-05239-7_1

[27] Ashby, N. (2002) Relativity and the Global Positioning System. Physics Today, 55, 41-47. http://www.livingreviews.org/lrr-2003-1 https://doi.org/10.1063/1.1485583

[28] Langley, R.B. (2000) GPS, the Ionosphere, and the Solar Maximum. GPS World, 11, 44-49.

[29] Golubkov, G.V., Manzhelii, M.I. and Eppelbaum, L.V. (2018) Quantum Theory of Disturbance and Delay of GPS Signals in $D$ and $E$ Atmospheric Layers: An Introduction. Positioning, 9, 13-22. https://doi.org/10.4236/pos.2018.92002

[30] Buenker, R.J., Golubkov, G.V., Golubkov, M.G., et al. (2013) Relativity Laws for the Variation of Rates of Clocks Moving in Free Space and GPS Positioning Errors Caused by Space-Weather Events. In: Mohamed, A.H., Ed., Global Navigation Satellite Systems-From Stellar to Satellite Navigation, INTECH, Amsterdam, London, New York, 1-48.

[31] Klobuchar, J. (1987) Ionospheric Time-Delay Algorithms for Single-Frequency GPS users. IEEE Transactions on Aerospace and Electronic Systems, 3, 325-331. https://doi.org/10.1109/TAES.1987.310829

[32] Landau, L.D. and Lifshitz, E.M. (1984) Electrodynamics of Continuous Media. Pergamon Press, Oxford.

[33] Avakyan, S.V. (2008) Physics of the Solar-Terrestrial Coupling: Results, Problems, and New Approaches. Geomagnetism and Aeronomy, 48, 417-424. https://doi.org/10.1134/S0016793208040014

[34] Golubkov, G.V., Golubkov, M.G. and Ivanov, G.K. (2010) Rydberg States of Atoms and Molecules in a Field of Neutral Particles. In: Bychkov, V.L., Golubkov, G.V. and Nikitin, A.I., Eds., The Atmosphere and Ionosphere: Dynamics, Processes and Monitoring, Springer, New York, 1-67. https://doi.org/10.1007/978-90-481-3212-6_1

[35] Jacobsen, K.S., Pedersen, A., Moen, J.I., et al. (2010) A New Langmuir Probe Concept for Rapid Sampling of Space Plasma Electron Density. Measurement Science \& Technology, 21, Article ID: 085902. https://doi.org/10.1088/0957-0233/21/8/085902

[36] Rurihara, J., Abe, T., Oyama, K., et al. (2006) Observation of the Lower Thermospheric Neutral Temperature and Density in the DELTA Campaign. Earth, Planets and Space, 58, 1123-1130. https://doi.org/10.1186/BF03352001

[37] Oyama, K.I., Abe, T., Mori, H. and Liu, J.Y. (2008) Electron Temperature in Nighttime Sporadic E Layer at Mid-Latitude. Annales Geophysicae, 26, 533-541.

https://www.ann-geophys.net/26/533/2008/Ann.Ge

https://doi.org/10.5194/angeo-26-533-2008 
[38] Golubkov, G.V. and Ivanov, G.K. (2001) Nonadiabatic Effects in Rydberg Molecular States and Their Manifestation in Collision Processes. Chemical Physics Reports, 19, 549-571.

[39] Mlynczak, M.G., Johnson, D.G., Latvakovski. H., et al. (2006) First Light from the Far-Infrared Spectroscopy of the Troposphere (FIRST) Instrument. Geophysical Research Letters, 33, L07704. https://doi.org/10.1029/2005GL025114

[40] Golubkov, G.V., Manzhelii, M.I., Berlin, A.A. and Lushnikov, A.A. (2014) Fundamentals of Radio-Chemical Physics of the Earth's Atmosphere. Russian Journal of Physical Chemistry B, 10, 77-90. https://doi.org/10.1134/S1990793116010024

[41] Berestetskii, V.B., Lifshitz, E.M. and Pitaevskii, L.P. (1982) Quantum Electrodynamics. Pergamon Press, New York.

[42] Golubkov, G.V. and Golubkov, M.G. (2014) Perturbation of Highly Excited States of an Atom by the Field of a Neutral Particle. Russian Journal of Physical Chemistry B, 8, 35-44. https://doi.org/10.1134/S1990793114010114

[43] Golubkov, G.V. and Ivanov, G.K. (2001) Rydberg States of Atoms and Molecules and Elementary Processes with their Participation. URSS, Moscow.

[44] Biberman, L.M., Vorob'ev, V.S. and Yakubov, I.T. (1987) Kinetics of Nonequilibriun Low-Temperature Plasmas. Springer, New York. https://doi.org/10.1007/978-1-4684-1665-7

[45] Svanberg, S. (2001) Atomic and Molecular Spectroscopy: Basic Aspects and Practical Applications. Springer, New York. https://doi.org/10.1007/978-3-642-98107-4

[46] Golubkov, G.V., Devdariani, A.Z. and Golubkov, M.G. (2002) Collision of Rydberg Atom $A^{\star *}$ with Atom B in the Ground Electronic State. Optical Potential. Journal of Experimental and Theoretical Physics, 95, 987-997. https://doi.org/10.1134/1.1537291

[47] Golubkov, G.V. and Ivanov, G.K. (1984) Near-Threshold Photoionization Theory for Diatomic Molecules. Journal of Physics B: Atomic and Molecular Physics, 17, 747-761. https://doi.org/10.1088/0022-3700/17/5/016

[48] Balashov, E.M., Golubkov, G.V. and Ivanov, G.K. (1984) Radiative Transitions between Rydberg States of Molecules. Journal of Experimental and Theoretical Physics, 59, 1188-1194.

[49] Lushnikov, A.A. and Maksimenko, V.V. (1993) Quantum Optics of a Metallic Particle. Journal of Experimental and Theoretical Physics, 76, 497-513. 